\title{
CANCER
}

\section{Lethal cycling}

Finding the Achilles heel of specific cancer types is a major goal in the development of targeted therapies. Now, recent evidence indicates that a new synthetic lethal strategy could be useful for oncogenic mutant KRAS-driven lung cancer.

Following their genetic identification in yeast, cyclin-dependent kinases (CDKs) were thought to be essential, ubiquitous drivers of

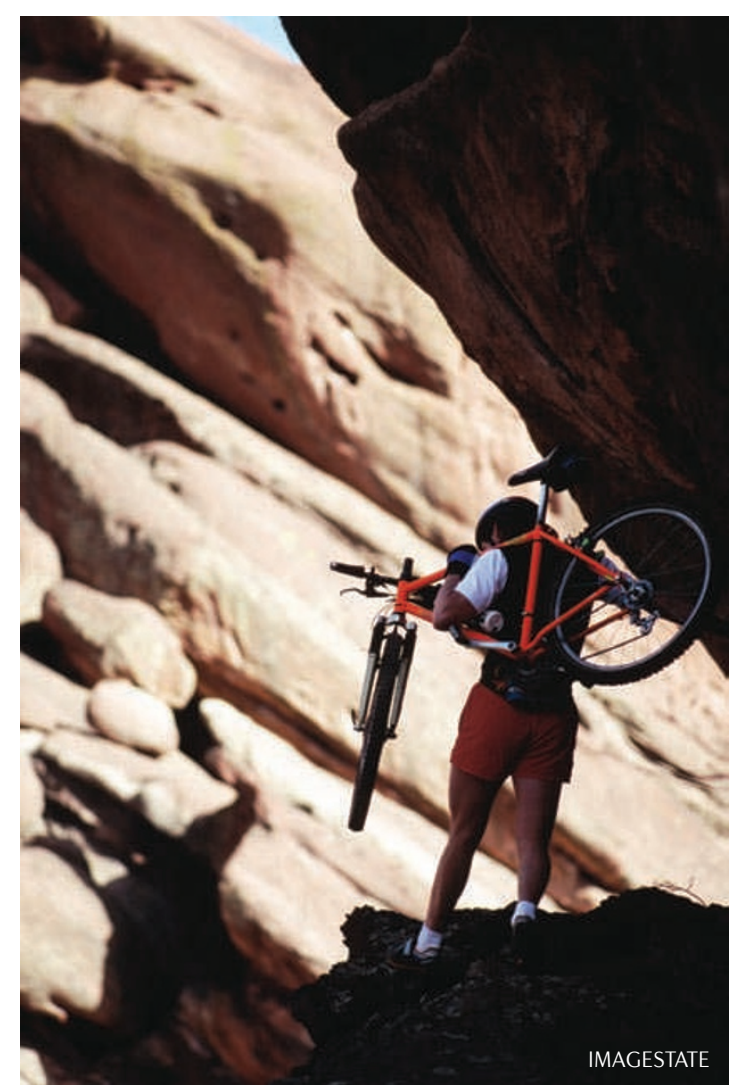

cell cycle progression. Although this remains true for the mitotic CDK (CDK1), knockout mouse studies showed that the interphase CDKs (CDK2, CDK4 and CDK6) are dispensable for viability and are only required for specialized, tissue-specific roles. This has raised questions about the validity of CDKtargeting agents as anti-proliferative therapies.

A team led by Mariano Barbacid examined the dependence on interphase CDKs of lung cancer driven by KRAS-G12V, a frequent lesion in human cancers of the lung, pancreas and colon. In vitro experiments using CDK-knockout mouse embryo fibroblasts (MEFs) revealed that deletion of $C d k 2$, $C d k 4$ or $C d k 6$ prevents the growth of KRAS-G12V-expressing MEFs in low-serum conditions and that knock down of any of these CDKs in human lung cancer cell lines is selectively lethal in KRAS-G12 mutant versus KRAS wild-type cells. However, follow-up in vivo studies in germline $C d k 2$ - or Cdk6-knockout mice failed to prevent KRAS-G12V-induced lung tumorigenesis. By contrast, deletion of $C d k 4$ resulted in marked senescence of KRAS-G12V-expressing preneoplastic lung cells, blocking their proliferation and preventing the development of high-grade lung tumours in vivo. Moreover, the loss of CDK4 function by either genetic excision or pharmacological inhibition in mice with established KRAS-G12V lung tumours significantly slowed tumour progression, providing further evidence of the therapeutic potential of this approach and implicating CDK4 in both the initiation and progression of KRAS-G12V-induced lung tumours. Interestingly, although KRAS-G12V expression was also induced in other tissues of the mouse, such as the pancreas and colon, these tissues do not undergo hyperplasia or develop tumours, and senescence was not induced in the absence of CDK4. This suggests that the induction of senescence after CDK4 loss could be specific to aberrantly proliferating tissues and so might be selective for tumour cells.

This work shows that certain oncogenic mutations can increase the cellular dependence on the interphase CDKs, thus renewing interest in CDK-targeted therapies. However, the success of these drugs in the clinic will depend on the context of the tumour tissue type and the specific driving oncogenes. Finally, it will be interesting to see whether this response to CDK4 inhibition will be sufficient to observe clinical responses in patients with oncogenic KRAS-mutant tumours.

Darren J. Burgess

ORIGINAL RESEARCH PAPER Puyol, M. et al A synthetic lethal interaction between K-Ras oncogenes and $\mathrm{Cdk} 4$ unveils a therapeutic strategy for non-small cell lung carcinoma. Cancer Cell 18, 63-73 (2010) 